\title{
Description and application of the background irradiance gradient-single turnover fluorometer (BIG-STf)
}

\author{
Zackary I. Johnson* \\ Massachusetts Institute of Technology (MIT), Department of Civil and Environmental Engineering, \\ 77 Massachusetts Avenue, Cambridge, Massachusetts 02139-4307, USA
}

\begin{abstract}
Based on previous single turnover, pulse amplitude modulated, and fast repetition rate fluorometers, I describe a novel, bench top, single turnover fluorometer (BIG-STf) that quantifies multiple biophysical properties of Photosystem II (PSII) of phytoplankton over a programmable range of background light levels. The instrument measures the photochemical conversion efficiency (variable fluorescence/maximal fluorescence yield, $\left.F_{\mathrm{v}} / F_{\mathrm{m}}\right)$ and functional cross-sectional area of PSII $\left(\sigma_{\text {PSII }}\right)$ over a background light gradient, and generates light-response curves of the biophysical properties of PSII. These curves can be used to assess variability in PSII structure and function or, in conjunction with oxygen- or carbon-derived photosynthesis-irradiance $(P-E)$ curves, to evaluate how the properties of PSII may influence total photosynthetic rate and efficiency. Nitrogen-starved batch cultures of Skeletonema costatum are used to demonstrate the utility of these measurements by comparing the quantum yield of carbon uptake as a function of light $\left(\phi_{\mathrm{C}}-E\right)$ and its saturation intensity index $\left(E_{k, \phi}\right)$ to $F_{\mathrm{v}} / F_{\mathrm{m}}$ and $\sigma_{\mathrm{PSII}}$ as a function of light $\left(F_{\mathrm{v}} / F_{\mathrm{m}}-E\right.$ and $\left.\sigma_{\mathrm{PSII}}-E\right)$ and their saturation intensity indices $\left(E_{k, \text { PSII }}\right)$. It is shown that in addition to changes in dark-measured values $\left(\phi_{\mathrm{C}, \max }, F_{\mathrm{v}} / F_{\mathrm{m}(0)}\right.$ and $\left.\sigma_{\mathrm{PSII}(0)}\right)$ there are also significant changes in the shapes of $\phi_{\mathrm{C}}-E_{1} F_{\mathrm{v}} / F_{\mathrm{m}}-E$ and $\sigma_{\mathrm{PSII}}-E$ curves in response to $\mathrm{N}$ starvation. Changes in the shape of the curves (summarized by approximate 2-fold decreases in both $E_{k, \phi}$ and $\left.E_{k, P S I I}\right)$ are consistent with the observed $\sim$-fold increase in $\sigma_{\text {PSII }}\left(r^{2}=0.74\right)$. These results suggest that in response to $N$ starvation (1) most of the decrease in $\phi_{C}$ can be explained by PSIIdependent processes, $(2)$ there are decreased saturation intensities of $F_{\mathrm{v}} / F_{\mathrm{m}}$ and $\phi_{\mathrm{C}}$ with concomitant increases in $\sigma_{\mathrm{PSI}(0)}$, and (3) the turnover rate $(1 / \tau)$ does not change significantly. The PSII lightresponse curves, which can be measured quickly by the BIG-STf instrument, provide a direct means of evaluating the role that PSII plays in regulating photosynthetic rates and efficiency in aquatic environments.
\end{abstract}

KEY WORDS: Fluorescence · Photosystem II · Instrumentation · Fluorometer · Nitrogen starvation · Skeletonema costatum

Resale or republication not permitted without written consent of the publisher

\section{INTRODUCTION}

An understanding of the variability of photosynthetic rates and efficiency of phytoplankton communities is an important step in describing how marine ecosystems are structured and function; yet current estimates of primary production using remotely sensed data are limited by photosynthetic efficiency terms, which can be parameterized in numerous and sometimes contradictory ap- proaches (Behrenfeld \& Falkowski 1997, Campbell et al. 2002). Direct comparisons between environmental variables such as nutrients, temperature and taxonomic composition and photosynthetic efficiency have also yielded relatively poor correlations (Johnson \& Howd 2000, Johnson et al. 2002). These simple relationships between photosynthetic efficiency and potentially important environmental variables may be inadequate to understand variations in photosynthetic efficiency. 
One approach to understand the variations in photosynthetic efficiency is to develop techniques to separate the components of the photosynthetic response. Variability in the individual components may offer insight into how environmental variables influence specific portions of the overall response, thus leading to a better understanding of the system as a whole (Geider \& Osborne 1992, Falkowski \& Raven 1997). For example, although temperature and taxonomic composition are not necessarily directly correlated with the maximum quantum yield of photosynthesis, a measure of photosynthetic efficiency, these environmental variables can be highly correlated with components of the photosynthetic process such as absorption properties or photosynthetic unit turnover rate (Schofield et al. 1993, Johnsen et al. 1994, Gibb et al. 2000). These parameters, in conjunction with others, can then be used with considerable fidelity to reconstruct how environmental factors may influence overall photosynthetic efficiency (Behrenfeld et al. 2002a,b).

To describe some of the individual component processes of photosynthesis, active phytoplankton fluorescence techniques-using pump-and-probe, pulse amplitude modulated or fast repetition rate fluorometers - provide a methodology for rapidly assessing the properties of Photosystem II (PSII) (Schreiber et al. 1989, Kolber \& Falkowski 1993, Kolber et al. 1998). These active fluorescence instruments can simultaneously make multiple measures of the structure and function of PSII, providing a more detailed assessment of the machinery of photosynthesis than total photosynthetic rate measurements alone (Kolber et al. 1998). PSII is of particular interest because it has been demonstrated to be specifically affected by multiple environmental variables such as nitrogen concentration, iron concentration and excess light (Kolber et al. 1988, Greene et al. 1992, Long et al. 1994, Vassiliev et al. 1994, Baroli \& Melis 1998) and these factors may be responsible for a large fraction of the variability of photosynthetic efficiency in marine environments (Falkowski \& Kolber 1995, Falkowski 1997, Behrenfeld \& Kolber 1999). Thus, properties and processes associated with PSII are a good initial target for dissecting the total photosynthetic response, and properties of PSII have been shown to be good indicators of photosynthetic responses in marine environments (Boyd et al. 1997).

Although previous studies describing the linkage between PSII and photosynthetic rates/efficiency or environmental parameters have been quite successful, they have focused on the properties of PSII measured in the dark (i.e. no background light) (Kolber et al. 1988, Babin et al. 1996, Boyd et al. 1997, Parkhill et al. 2001). Because properties of PSII, such as the photosynthetic conversion efficiency $\left(F_{\mathrm{v}} / F_{\mathrm{m}}\right)$ and functional cross-sectional area $\left(\sigma_{\text {PSII }}\right)$, are critically dependent on the light-regulated oxidation state of photosynthetic reaction centers and the photosynthetic electrontransport chain (Falkowski et al. 1986, Olaizola et al. 1994), environmentally induced changes in dark-measured $F_{\mathrm{v}} / F_{\mathrm{m}}$ and $\sigma_{\mathrm{PSII}}$ may only be partially diagnostic of the influence that PSII properties have on photosynthetic efficiency in the light. Describing how $F_{\mathrm{v}} / F_{\mathrm{m}}$ and $\sigma_{\text {PSII }}$ vary as a function of light intensity by constructing so-called light-response curves $\left(F_{\mathrm{v}} / F_{\mathrm{m}}-E_{1} \sigma_{\mathrm{PSII}}-E\right)$, which are operationally similar to photosynthesisirradiance $(P-E)$ curves, may provide additional insight into how environmental factors can influence PSII and, ultimately, photosynthetic efficiency.

Thus, the aim of this study was (1) to describe the design and function of the background irradiance gradient-single turnover fluorometer (BIG-STf), a novel fluorometer that is capable of quickly measuring multiple, single turnover, fluorescence induction curves over a light gradient, and (2) to demonstrate how the parameters derived from these curves can vary under a controlled situation that is known to affect PSII (nitrogen starvation). I show that, consistent with previous findings of nitrogen starvation, the BIG-STf measures a significant alteration in the dark-measured parameters of $F_{\mathrm{v}} / F_{\mathrm{m}}$ and $\sigma_{\mathrm{PSII}}$. I also show that the shape of the light-response curves $\left(F_{\mathrm{v}} / F_{\mathrm{m}}-E\right.$ and $\left.\sigma_{\mathrm{PSII}}-E\right)$, which is summarized by a saturation parameter, changes significantly in response to $\mathrm{N}$ starvation. Both of these results are consistent with and help to describe the patterns observed for the quantum yield of carbon uptake $\left(\phi_{\mathrm{C}}\right)$. Together these results demonstrate the utility of the BIG-STf instrument to describe and interpret changes in the properties of PSII, which may in turn lead to a more detailed understanding of the variability of photosynthesis in marine ecosystems.

\section{MATERIALS AND METHODS}

Nitrogen-starved cultures. Replicate Skeletonema costatum cultures were grown in batch culture in 51 Pyrex containers at $19^{\circ} \mathrm{C}$ in air-bubbled, modified $\mathrm{f} / 2$ media (N/20 and $1 \mathrm{Si}$, with major plant-nutrient, atom ratios of N:P:Si 2.4:1:5.8) (Guillard \& Ryther 1962). Assuming balanced Redfield growth (N:P:Si 16:1: 10.4 to 15.0), nitrogen is predicted to limit growth first (Redfield 1958, Brzezinski 1985). Nominal nitrogen starvation was relieved by addition of $f / 2$ concentrations of nitrogen stock solution $(0.88 \mathrm{mM} \mathrm{N}$, final concentration). Continuous light $\left(\sim 100 \mu \mathrm{mol}\right.$ quanta $\mathrm{m}^{-2}$ $\mathrm{s}^{-1}$ ) was supplied by cool white fluorescent bulbs. Cultures were started and remained optically thin (mean $\mathrm{OD}<0.1 \mathrm{~cm}^{-1}$ ) throughout the duration of the experiment. Growth rate was determined from chlorophyll $a$ 
biomass measured using standard spectrophotometric techniques (Parsons et al. 1984).

Absorption, photosynthesis-irradiance curves, and maximum quantum yield of carbon uptake. Spectral absorption measurements were made at $2 \mathrm{~nm}$ resolution, with a $1 \mathrm{~s}$ integration time, on samples in solution, using an HP 8452 diode-array spectrophotometer with Labsphere (RSA-HP-84) integrating sphere. Absorption at $750 \mathrm{~nm}$ was assumed to be non-cellular and was subtracted from the absorption curve. Absorption coefficients were calculated after Kirk (1994). Radiocarbon-based photosynthesis-irradiance (P-E) measurements were made using custom-built temperature-regulated photosynthetrons that have 13 positions, each at a different light level (Johnson et al. 2002). Briefly, irradiance, which was supplied by a 250 W ENH projector bulb (Gray Supply), was spectrally modified and attenuated using a combination of hot and cold mirrors (Optical Coating Laboratory) and neutral-density screening (Cinemills). Photosynthetically active radiation (PAR) was measured using a Biospherical Instruments (QSL-100) $4 \pi$ scalar irradiance meter. Incubations of $1 \mathrm{ml}$ samples inoculated with $\sim 7.4 \mathrm{kBq} \mathrm{H}{ }^{14} \mathrm{CO}_{3}$ were terminated after $10 \mathrm{~min}$ using $100 \mu \mathrm{l} 37 \%$ formaldehyde and $200 \mu \mathrm{l} 1 \mathrm{~N} \mathrm{HCl}$ and allowed to degas overnight. Carbon uptake rates were quantified using standard techniques (Barber et al. 1996). The quantum yield of carbon uptake for each measurement light level was calculated after Johnson \& Barber (2003).

Modulated fluorescence (BIG-STf). Modulated fluorescence measurements were made with a custom-built single turnover fluorometer with computer-controlled background irradiance gradient (BIG-STf). This instrument is based on a ring of super-bright blue LEDs (Nichia NSPB500S, maximum emission $470 \mathrm{~nm}$, spectrum half-width $30 \mathrm{~nm}$ ) that illuminate an aqueous sample of phytoplankton producing fluorescence (Fig. 1): a bank of LEDs (84 in total) is operated in flash mode (maximum frequency of $1 \mathrm{MHz}$ ) and is used to excite and probe the response of PSII to a single turnover flash; a second bank of LEDs (28 in total) is operated in continuous mode and is used to provide a constant background irradiance source, which can be set from 0 to $\sim 1000 \mu \mathrm{mol}$ quanta $\mathrm{m}^{-2} \mathrm{~s}^{-1}$. The intensity of the continuous light source was quantified using a Biospherical Instruments (QSL-100) $4 \pi$ scalar irradiance meter. The red fluorescence signal $(\sim 680 \mathrm{~nm})$ is isolated through a long-pass dichroic (Melles-Griot \#RG665) and interference (Oriel \#53970) filter set and quantified by means of a photomultiplier tube (Hamamatsu \#R2066). A fraction of the excitation energy is quantified with a PIN photodiode (Hamamatsu \#S1722-02). Excitation and emission signals are simultaneously sampled at $10 \mathrm{MHz}$

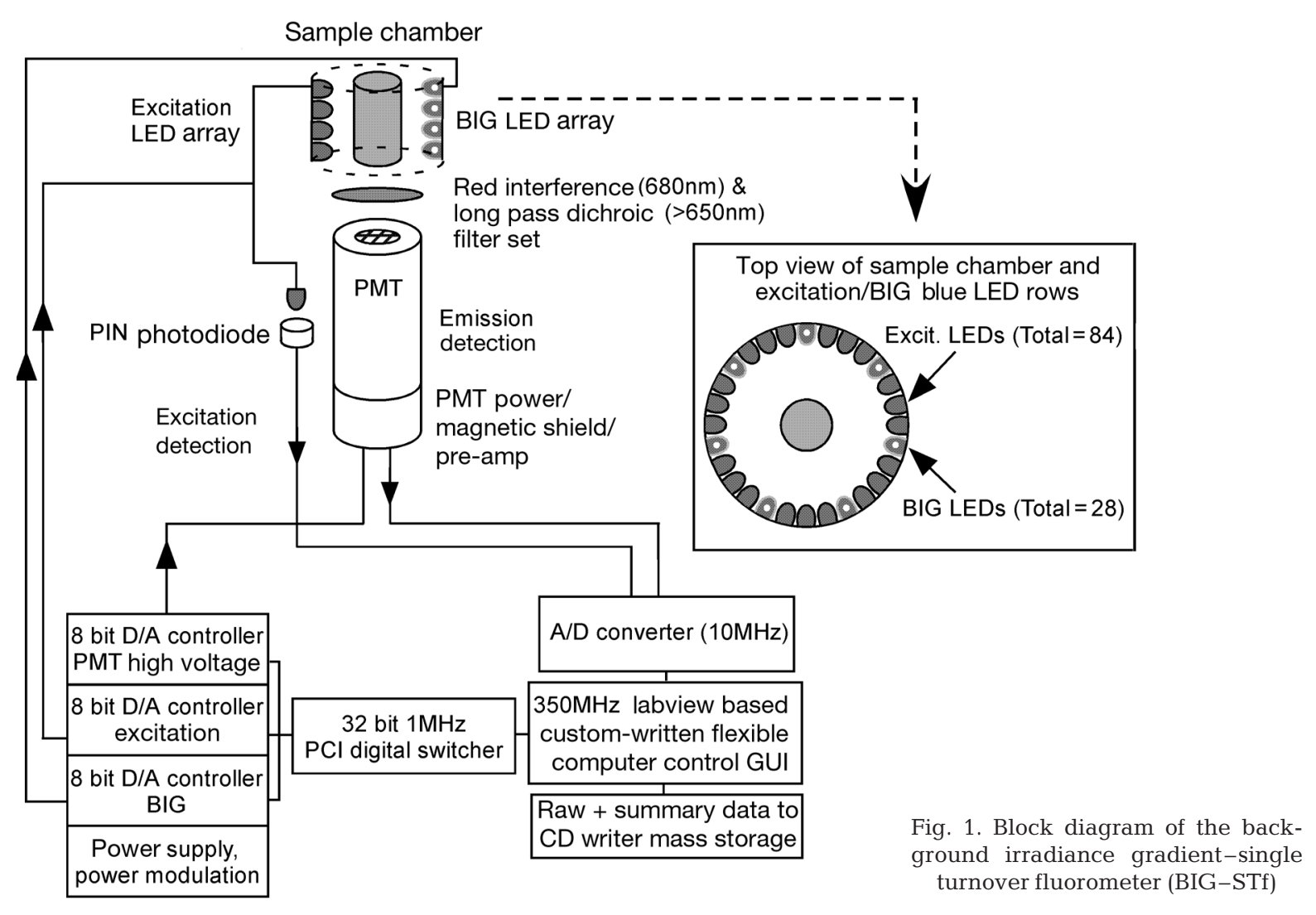


Typical ST-fluorescence induction curve

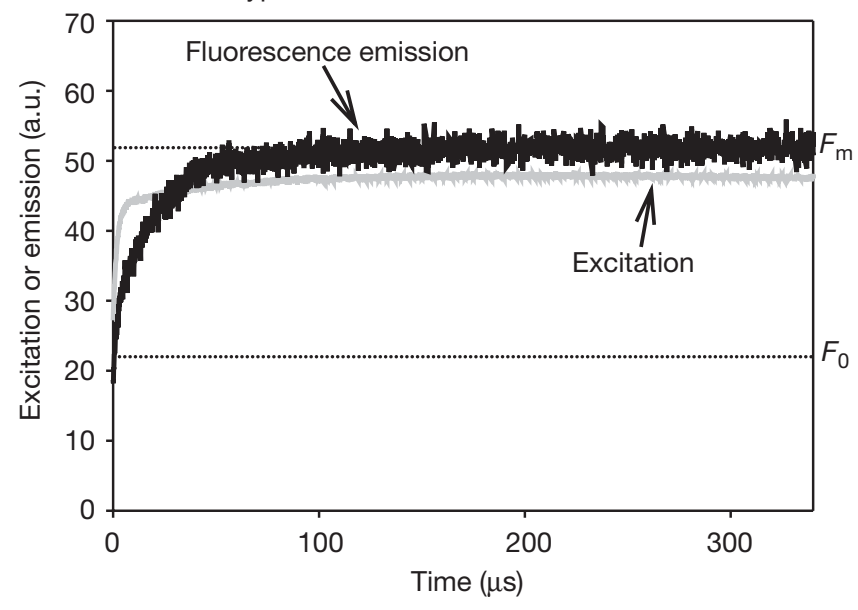

Fig. 2. Example of excitation and single turnover (ST) fluorescence emission curves used to generate fluorescence-yield curves. $F_{0}$ : initial fluorescence; $F_{\mathrm{m}}$ : maximal fluorescence; a.u.: arbitrary units

and passed through an analog-to-digital converter (Gage Compuscope 1012PCI).

The measurement protocol is computer-controlled by custom-written software via a graphical user interface that allows substantial flexibility in the protocol.

Samples were dark-acclimated for $>15$ min before the initiation of the background irradiance gradient. Data discussed here were generated using a preillumination background irradiance of $500 \mathrm{~ms}$ (which is sufficient for multiple turnovers of PSII under saturating irradiance; Falkowski \& Raven 1997) and a continuous excitation pulse $(100 \%$ duty cycle) of $350 \mu \mathrm{s}$. Analogous to standard oxygen-evolution techniques, each sample was exposed to the entire range of light levels, stepping from dark to high light (Walker 1988).

Representative raw data from the BIG-STf is presented in Fig. 2. Raw data are internally processed to isolate excitation and fluorescence signals from background noise. This data is converted into reduced fluorescence emission data by binning and averaging over $1 \mu$ s blocks and normalizing the signal to binned and averaged excitation data, producing a relative fluorescence yield. The frequency resolution of the reduced data is $1 \mathrm{MHz}$. Next, the fluorescence yield data is corrected for non-phytoplankton background fluorescence (as determined from filtered water blanks). Finally, a sample of extracted chlorophyll in $100 \%$ methanol is used as a control to ensure that the fluorescence induction curve for dissociated pigment molecules is a line with a slope of zero (i.e. $F_{\mathrm{v}} / F_{\mathrm{m}}=0$ ). This corrected signal produces a fluorescence yield curve that, along with a cumulative excitation curve, is subsequently analyzed.
Values of $F_{0}$ (initial fluorescence), $F_{\mathrm{m}}$ (maximal fluorescence) and $\sigma_{\mathrm{PSII}}$ were determined from single turn-over fluorescence-induction curves using a custom-written, non-linear least-squares LevenbergMarquardt technique following Kolber et al. (1998). $F_{\mathrm{v}}$ (variable fluorescence) is calculated as $F_{\mathrm{m}}-F_{0}$. When $F_{\mathrm{v}} / F_{\mathrm{m}}<0.075$, there is low statistical confidence in estimating $\sigma_{\text {PSII }}$ therefore, these values were excluded from further analyses. Although the quantum output of flashes was not directly quantified (and therefore values of $\sigma_{\mathrm{PSII}}$ are given as arbitrary units), saturation of PSII during fluorescence induction was verified by fluorescence approaching an asymptote before the turnover time (Fig. 3) (Kolber et al. 1998). Saturation intensity indices, $E_{k, F_{\mathrm{V}} / F_{\mathrm{m}}{ }^{\prime}} E_{k, \sigma \mathrm{\sigma PSII}}$ and $E_{k, \phi}$ which are similar to $E_{k}$ derived from $P-E$ curves (Geider \& Osborne 1992), were calculated by curve-fitting

$$
\begin{array}{ll}
{\left[\left(F_{\mathrm{v}} / F_{\mathrm{m}(0)}-C_{1}\right) \exp \left(-E / E_{k, F_{\mathrm{v}} / F_{\mathrm{m}}}\right)+C_{1}\right.} & \text { to } F_{\mathrm{v}} / F_{\mathrm{m}}-E_{1} \\
{\left[\left(\sigma_{\mathrm{PSII},(0)}-C_{2}\right) \exp \left(-E / E_{k, \sigma \mathrm{PSII}}\right)+C_{2}\right]} & \text { to } \sigma_{\mathrm{PSII}}-E_{1}
\end{array}
$$

and

$$
\left[\left(\phi_{C, \max }-C_{3}\right) \exp \left(-E / E_{k, \phi}\right)+C_{3}\right] \quad \text { to } \phi_{\mathrm{C}}-E
$$

In these equations, $C_{\mathrm{x}}$ is a constant that indicates the non-zero asymptote of the curve. Values of $\phi_{C}$ made at light levels below the light level at which $\phi_{\mathrm{C}, \max }$ occurs were not included in the curve fit (Johnson \& Barber 2003). The photosynthetic unit turnover rate, $(1 / \tau)$ was calculated as:

$$
1 / \tau=E_{k, \mathrm{PSII}} \sigma_{\mathrm{PSII}}
$$

(Falkowski \& Raven 1997).

ST-fluorescence induction curves and background light

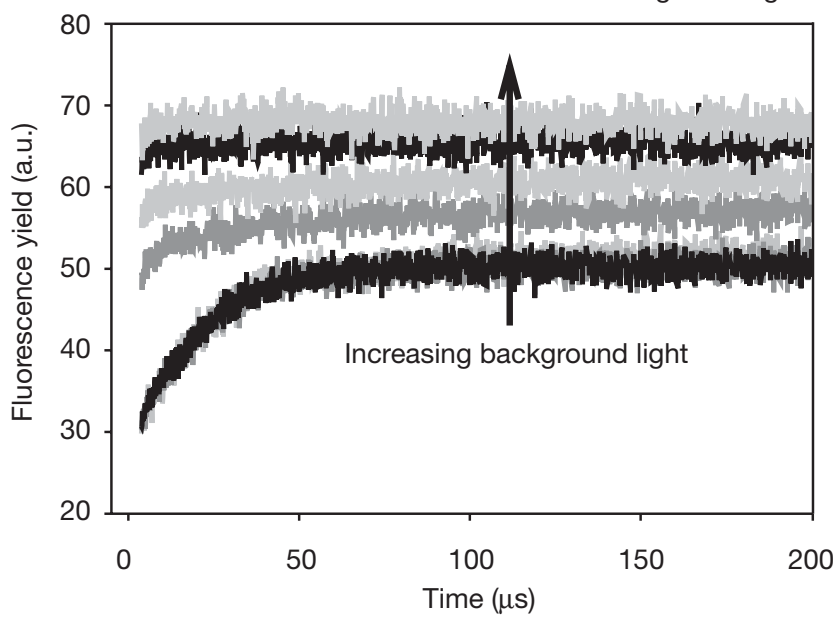

Fig. 3. Example of a family of single turnover (ST) fluorescence induction curves from which values of $F_{0}, F_{\mathrm{m}}$ and $\sigma_{\mathrm{PSII}}$ (cross-sectional area of PSII) were derived. Each curve corresponds to a different background light level, with increasing light levels resulting in increased $F_{0}$ and $F_{\mathrm{m}}$ 
At each background light level, a single turnover, fluorescence induction curve is generated, from which a suite of fluorescence-derived parameters is derived (Fig. 3). These parameters can be plotted as a function of irradiance-yielding light-response curves for the various parameters (Fig. 4). Following plant physiology convention (van Kooten \& Snel 1990), parameters measured in the presence of background light have a superscript ('). Additionally, because numerous background light levels are used in this study, parameters are referred to with a subscript indicating the background irradiance intensity. Thus, $F_{\mathrm{v}} / F_{\mathrm{m}(0)}$ is PSII photochemical conversion efficiency in the dark, and $\sigma_{\text {PSII(521) }}^{\prime}$ is the PSII functional cross-sectional area at $521 \mu \mathrm{mol}$ quanta $\mathrm{m}^{-2} \mathrm{~s}^{-1}$. Other parameters not presented here, such as non-photochemical quenching (NPQ) and photosystem connectivity $(p)$, can also be derived from these curves (Demmig-Adams et al. 1996, Kolber et al. 1998).

\section{RESULTS AND DISCUSSION}

\section{Instrumentation and light-response curves}

The BIG-STf instrument was designed to bridge the gap between the capabilities of existing commercially available single turnover and multiple turnover fluorometers by measuring single turnover fluorescence induction curves in the presence of a range of background light levels. Pump-and-probe type fluorometers, which measure the fluorescence before and after a single turnover saturating pulse, have been used to measure $F_{\mathrm{v}} / F_{\mathrm{m}}$ over a light gradient, but do not measure $\sigma_{\text {PSII }}$ (Falkowski et al. 1986). Commercially available pulse amplitude modulated (PAM) fluorometers, which continually measure fluorescence before and after multiple-turnover flashes, are routinely used to measure $F_{\mathrm{v}} / F_{\mathrm{m}}$ in the presence of background light, but again do not measure $\sigma_{\mathrm{PSII}}$ (Schreiber et al. 1986). Commercially available fast repetition rate fluorometers (FRRf), which simultaneously measure $F_{\mathrm{v}} / F_{\mathrm{m}}$ and $\sigma_{\text {PSII }}$ by pulsing with sub-saturating flashlets that produce a single turnover, fluorescence induction curve (Kolber et al. 1998, www.chelsea.co.uk), are designed for in situ application and do not allow the regulation of background light levels. The BIG-STf instrument, which is a bench-top fluorometer, produces single turnover, fluorescence induction curves like the FRRf, and therefore measures both $F_{\mathrm{v}} / F_{\mathrm{m}}$ and $\sigma_{\mathrm{PSII}}$. However, since background light levels can also be controlled, both $F_{\mathrm{v}} / F_{\mathrm{m}}$ and $\sigma_{\mathrm{PSII}}$ light-response curves can be constructed. These curves allow the direct assessment of how PSII affects photosynthesis over a range of light levels. Since light varies significantly in the upper

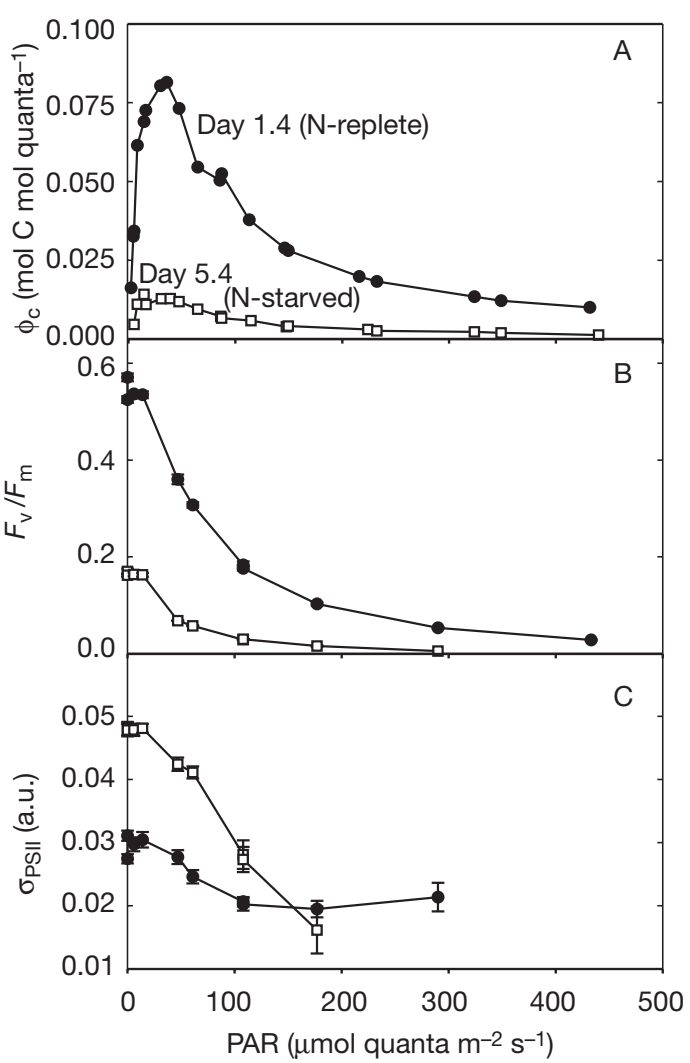

Fig. 4. Skeletonema costatum. Representative light-response curves measured on nitrogen-starved batch culture (A) $\phi_{\mathrm{C}}-E_{\text {; }}$ (B) $\left.F_{\mathrm{v}} / F_{\mathrm{m}}-E_{\mathrm{i}}(\mathrm{C}) \sigma_{\mathrm{PSII}}-E\right)$. Day 1.4 curves $(\bullet)$ nitrogen-replete, Day 5.4 curves ( $\square$ ) are nominally nitrogen-starved

ocean and PSII is specifically affected by environmental variables (Kolber et al. 1988, Kirk 1994, Babin et al. 1996), understanding how PSII responds over a light gradient is an important step in understanding what regulates primary production and photosynthetic efficiency in the open ocean.

\section{Nitrogen starvation}

To demonstrate how the properties of PSII might vary over a light gradient, cultures of Skeletonema costatum were nitrogen-starved; this has previously been shown to significantly affect PSII (Kolber et al. 1988, Parkhill et al. 2001). Nitrogen starvation significantly affected the biomass properties and growth rate of the $S$. costatum cultures, and the initial exponential growth of the culture $\left(\sim 1.5 \mathrm{~d}^{-1}\right)$ declined steadily in response to $\mathrm{N}$ starvation until, by Day 2, the growth rate was nearly zero and the biomass and physiological properties began to change (Johnson 2000) (Fig. 5). These changes were reversed by the addition of nitro- 


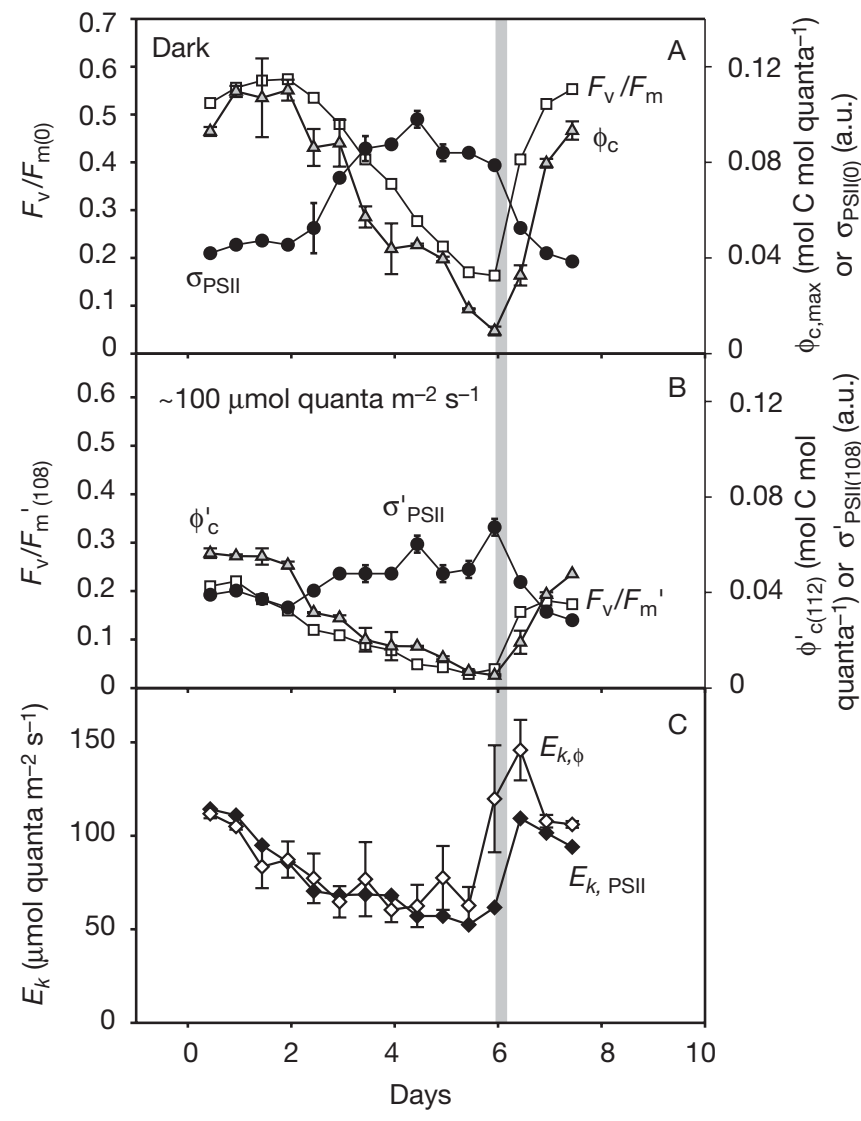

Fig. 5. Skeletonema costatum. Time course for nitrogenstarved batch cultures. (A) Dark-measured photosynthetic parameters. (B) Parameters measured at light level of $\sim 100 \mu \mathrm{mol}$ quanta $\mathrm{m}^{-2} \mathrm{~s}^{-1}$. (C) Saturation intensity indices, $E_{k}$. 口: $F_{\mathrm{v}} / F_{\mathrm{m} i} \bullet: \sigma_{\mathrm{PSII}} \boldsymbol{\Delta}: \phi_{\mathrm{C} i} \diamond: E_{k, \phi i} \bullet: E_{k, \text { PSII }}$ Nitrogen, from $\mathrm{f} / 2$ stock solution, was amended in cultures on Day 5.9 to final concentration of $0.88 \mathrm{mM} \mathrm{N}$ (vertical grey bar; see 'Results and discussion')

gen on Day 6 of the experiment, with most biomass and physiological parameters returning to pre-starvation values by the end of the experiment on Day 8 .

Under N-replete conditions, both the maximum quantum yield of carbon uptake $\left(\phi_{\mathrm{C}, \mathrm{max}}\right)$ and photochemical conversion efficiency of PSII $\left(F_{\mathrm{v}} / F_{\mathrm{m}(0)}\right)$ were near maximum at $0.082 \mathrm{~mol} \mathrm{C} \mathrm{mol} \mathrm{quanta}{ }^{-1}$ and 0.57 , respectively (Figs. 4 \& 5). Both these properties dramatically decreased in response to $\mathrm{N}$ starvation to 0.013 mol C mol quanta ${ }^{-1}$ and 0.17 , respectively, and are consistent with previous findings (Kolber et al. 1988, Parkhill et al. 2001). Concomitant with the decreases in photosynthetic yield and efficiency were increases $(\sim 80 \%)$ in $\sigma_{\mathrm{PSII}(0)}$, which were higher than in previous N-limitation studies, but still consistent with the direction of change (Kolber et al. 1988, Geider et al. 1993). Together, these results are consistent with previous findings of $\mathrm{N}$ starvation affecting photophysiol- ogy specifically through alterations in PSII by reducing or eliminating the ability to repair damaged PSII reaction centers and ultimately resulting in reduced photosynthetic efficiency (Kolber et al. 1988, Parkhill et al. 2001). Alterations in bulk pigmentation and the number of functional reaction centers in turn lead to $\mathrm{N}$ starvation affecting $\sigma_{\text {PSII(0) }}$ (Geider et al. 1993, 1998).

In addition to the changes induced by $\mathrm{N}$ starvation in dark-measured values of photosynthetic parameters $\left(F_{\mathrm{V}} / F_{\mathrm{m}(0)}\right.$ and $\left.\sigma_{\mathrm{PSII}(0)}\right)$, there were also significant responses in photosynthetic parameters in the presence of background light (Figs. 4 \& 5). For example, under $\mathrm{N}$ starvation near the growth irradiance of $\sim 100 \mu \mathrm{mol}$ quanta $\mathrm{m}^{-2} \mathrm{~s}^{-1}$ there were large decreases in $\phi_{\mathrm{C}(112)}^{\prime}$ (from 0.040 to $0.006 \mathrm{~mol} \mathrm{C} \mathrm{mol} \mathrm{quanta}{ }^{-1}$ ) and $F_{\mathrm{v}} / F_{\mathrm{m}}{ }^{\prime}(108)$ (from 0.18 to 0.03 ) and a $33 \%$ increase in $\sigma_{\text {PSII(108) }}^{\prime}$ (Fig. 5). Both dark-measured and light-measured photosynthetic parameters were tracked over the course of N starvation (Fig. 5) and showed similar patterns of reductions $\left(\phi_{\mathrm{C}}\right.$ and $\left.F_{\mathrm{v}} / F_{\mathrm{m}}\right)$ or increases $\left(\sigma_{\text {PSII }}\right)$. Other specific levels of background light showed similar patterns of reduction or increase, with $\phi_{\mathrm{C}}{ }^{\prime}$ and $F_{\mathrm{v}} / F_{\mathrm{m}}{ }^{\prime}$ highly correlated over all light levels $\left(\mathrm{r}^{2}=0.92\right)$. Together, these results demonstrate that under nitrogen starvation (1) the magnitude of change in the photosynthetic conversion efficiency of PSII $\left(F_{\mathrm{v}} / F_{\mathrm{m}}\right)$ is nearly identical to the total photosynthetic quantum yield $\left(\phi_{\mathrm{C}}\right)$ and is consistent with alterations to PSII, being responsible for the majority of the reduction in photosynthetic efficiency, and (2) the changes in light-measured parameters can be as significant as dark-measured parameters.

The changes in light-measured parameters were examined further by analyzing their component parts. Unlike responses in dark-measured parameters (e.g. $\left.\phi_{\mathrm{C}, \max }, F_{\mathrm{v}} / F_{\mathrm{m}(0)}\right)$, light-measured parameters are influenced by both the shape (response structure) and magnitude (typically the dark-measured value such as $\left.F_{\mathrm{v}} / F_{\mathrm{m}(0)}\right)$ of the light-response curve. To differentiate between these 2 effects, saturation intensity indices $\left(E_{k, \phi}, E_{k, F_{\mathrm{v}} / F_{\mathrm{m}}}\right.$ or $\left.E_{k, \sigma \text { PSII }}\right)$, which summarize the shape of these curves, were investigated. These saturation values, are inversely proportional to the functional absorption cross-section $\left(\sigma_{\mathrm{PSII}}\right)$ and directly proportional to the turnover rate $(1 / \tau)$ (Geider \& Osborne 1992). Values of $E_{k}$ derived from $F_{\mathrm{v}} / F_{\mathrm{m}}-E$ and $\sigma_{\mathrm{PSII}}-E$ curve-fits were nearly identical, (probably because they are both indicative of the saturation properties of PSII), and were therefore averaged, and are referred to as $E_{k, \text { PSII }}$ in further analyses. These results show that $\mathrm{N}$ starvation decreases $E_{k}$ by $\sim 50 \%$ from 113 to $60 \mu \mathrm{mol}$ quanta $\mathrm{m}^{-2} \mathrm{~s}^{-1}$ for both $F_{\mathrm{v}} / F_{\mathrm{m}}-E$ and $\phi_{\mathrm{C}}-E$ (Fig. 5C). Thus, under $\mathrm{N}$ starvation, photosynthetic yield and efficiency $\left(\phi_{\mathrm{C}}, F_{\mathrm{v}} / F_{\mathrm{m}}\right)$ are light-saturated at lower irradiances. 
The magnitude and timing of $E_{k}$ reduction for both $\phi_{\mathrm{C}}$ and PSII are similar (Fig. 5C), and the maximal values of $F_{\mathrm{v}} / F_{\mathrm{m}}\left(F_{\mathrm{v}} / F_{\mathrm{m}(0)}\right)$ and $\phi_{\mathrm{C}}\left(\phi_{\mathrm{C}, \max }\right)$ change similarly over the course of $\mathrm{N}$ starvation. Both $E_{k}$ and $F_{\mathrm{v}} / F_{\mathrm{m}(0)}$ or $\phi_{\mathrm{C}, \max }$ can influence parameters measured in the light. To determine the relative influence of these 2 components on light-measured values, a multiple linear regression was performed on natural logtransformed data $\left(\mathrm{r}^{2}=0.99\right)$. At a background light level of $\sim 100 \mu \mathrm{mol}$ quanta $\mathrm{m}^{-2} \mathrm{~s}^{-1}$ (the growth irradiance) the $\mathrm{N}$ starvation induced reduction in $E_{k}$ accounts for $\sim 55 \%$ of the reduction in $F_{\mathrm{v}} / F_{\mathrm{m}(108)}$, while the reduction in the $F_{\mathrm{v}} / F_{\mathrm{m}(0)}$ accounts for the remaining $\sim 45 \%$. Similarly, the reduction in $E_{k}$ accounts for $\sim 33 \%$ of the reduction in $\phi_{\mathrm{C}(112)}$, with the remaining variability explained by changes in $\phi_{\mathrm{C}(\max )}$. This result suggests that in response to $\mathrm{N}$ starvation, alterations to the shape of $F_{\mathrm{v}} / F_{\mathrm{m}}-E$ and $\phi_{\mathrm{C}}-E$ curves, as summarized by $E_{k}$, can be as important as changes in the magnitude (i.e. $F_{\mathrm{v}} / F_{\mathrm{m}(0)}$ and $\phi_{\mathrm{C}(\max )}$ ) in determining the total lightresponse curve. Furthermore, because the timing and levels of the reductions in $E_{k, P S I}$ and $E_{k, \phi}$ are similar, it is likely that the same process may be acting to change the shapes of both the $F_{\mathrm{v}} / F_{\mathrm{m}}-E$ and $\phi_{\mathrm{C}}-E$ curves.

By definition, changes in $E_{k}$ in response to $N$ starvation may be due to alterations in the functional absorption cross-section or turnover rate (Geider \& Osborne 1992). Using $\sigma_{\mathrm{PSII}}$ in conjunction with $E_{k}$ to calculate the turnover rate yields some variability in $1 / \tau$ (coefficient of variation $=20 \%$ ). However, unlike $E_{k}$ and $\sigma_{\text {PSII }}$, there is no pattern in $1 / \tau$ that is consistent with other biomass properties or parameters of photophysiology, and $74 \%$ of the variance in $E_{k}$ is explained by $\sigma_{\mathrm{PSII}(0)}$ suggesting that $1 / \tau$ plays a reduced role in $E_{k}$ variability. This is consistent with previous observations, which showed that although there are changes in some components of the photosynthetic electron transport chain in response to $\mathrm{N}$ starvation, other components remain less affected (Kolber et al. 1988). Thus, the majority of variability in $E_{k}$ in response to $\mathrm{N}$ starvation is probably due to changes in the functional absorption cross-section.

Taken together, the light-response curves of $F_{\mathrm{v}} / F_{\mathrm{m}}-E$ and $\sigma_{\mathrm{PSII}}-E$, in conjunction with $\phi_{\mathrm{C}}-E$ curves, demonstrate that $\mathrm{N}$ starvation leads to a similar reduction in $F_{\mathrm{v}} / F_{\mathrm{m}(0)}$ and $\phi_{\mathrm{C}, \max }$, suggesting a common mechanism for the reduction, namely damage to PSII reaction centers. In addition, at the growth irradiance (100 $\mu \mathrm{mol}$ quanta $\left.\mathrm{m}^{-2} \mathrm{~s}^{-1}\right), F_{\mathrm{v}} / F_{\mathrm{m}}{ }^{\prime}$ and $\phi_{\mathrm{C}}{ }^{\prime}$ are further reduced probably due to increases in the PSII functional absorption cross-section $\left(\sigma_{\mathrm{PSII}}\right)$, with relatively little variation in the turnover rate $(1 / \tau)$. This additional reduction is indicated by the decreases in saturation intensity indices for both $\phi_{\mathrm{C}}\left(E_{k, \phi}\right)$ and PSII $\left(E_{k, \mathrm{PSII}}\right)$.
The BIG-STf provides a rapid and non-destructive means of assessing multiple properties of PSII over a light gradient. In addition to measuring fundamental properties such as $F_{\mathrm{v}} / F_{\mathrm{m}(0)}$ and $\sigma_{\mathrm{PSII}(0)}$, these measurements can also provide information on how PSII properties change in response to light-dependent processes. For batch cultures of Skeletonema costatum that are N-starved, (a process that is known to strongly affect PSII), changes in the shape of the PSII lightresponse curves are as important as changes in the magnitude of those curves. The results demonstrate that the mechanisms which regulate the shape of lightresponse curves may be as important as those which determine their magnitudes for understanding how photosynthetic rates and efficiencies vary.

Acknowledgements. Z. J. would like to acknowledge Drs. R. T. Barber and P. Falkowski for discussions and the diligent comments of the reviewers. This work was supported by grants from NASA to R. T. B. and NSF to R. T. B. and S. W. Chisholm. Z. J. acknowledges assistance in instrument design from L. Borden and support from an NOAA Climate and Global Change postdoctoral fellowship.

\section{LITERATURE CITED}

Babin M, Morel A, Claustre H, Bricaud A, Kolber Z, Falkowski PG (1996) Nitrogen-and irradiance-dependent variations of the maximum quantum yield of carbon fixation in eutrophic, mesotrophic and oligotrophic marine systems. Deep-Sea Res Part I 43:1241-1272

Barber RT, Sanderson MP, Lindley ST, Chai F, Newton J, Trees CC, Foley DG, Chavez FP (1996) Primary productivity and its regulation in the equatorial Pacific during and following the 1991-1992 El Nino. Deep-Sea Res Part II 43:933-969

Baroli I, Melis A (1998) Photoinhibitory damage is modulated by the rate of photosynthesis and by the PSII lightharvesting chlorophyll antenna size. Planta 205:288-296

Behrenfeld MJ, Falkowski PG (1997) A consumer's guide to phytoplankton primary productivity models. Limnol Oceanogr 42:1479-1491

Behrenfeld MJ, Kolber ZS (1999) Widespread iron limitation of phytoplankton in the South Pacific Ocean. Science 283: 840-843

Behrenfeld M, Esaias W, Turpie K (2002a) Assessment of primary production at the global scale. In: Williams PJl, Thomas DN, Reynolds CS (eds) Phytoplankton productivity: carbon assimilation in marine and freshwater ecosystems, Blackwell Science, Oxford, p 156-186

Behrenfeld MJ, Maranon E, Siegel DA, Hooker SB (2002b) Photoacclimation and nutrient-based model of lightsaturated photosynthesis for quantifying oceanic primary production. Mar Ecol Prog Ser 228:103-117

Boyd PW, Aiken J, Kolber Z (1997) Comparison of radiocarbon and fluorescence based (pump and probe) measurements of phytoplankton photosynthetic characteristics in the Northeast Atlantic Ocean. Mar Ecol Prog Ser 149:215-226

Brzezinski MA (1985) The Si:C:N ratio of marine diatoms: interspecific variability and the effect of some environmental variables. J Phycol 21:347-357 
Campbell J, Antoine D, Armstrong R, Arrigo K and 19 others (2002) Comparison of algorithms for estimating ocean primary production from surface chlorophyll, temperature, and irradiance. Global Biogeochem Cycles 16:1035-1049

Demmig-Adams B, Adams WW, III, Barker DH, Logan BA, Bowling DR, Verhoeven AS (1996) Using chlorophyll fluorescence to assess the fraction of absorbed light allocated to thermal dissipation of excess excitation. Physiol Plant 98:253-264

Falkowski PG (1997) Evolution of the nitrogen cycle and its influence on the biological sequestration of $\mathrm{CO}_{2}$ in the ocean. Nature 387:272-275

Falkowski PG, Kolber Z (1995) Variations in chlorophyll fluorescence yields in phytoplankton in the world oceans. Australian J Plant Physiol 22:341-355

Falkowski P, Raven J (1997) Aquatic photosynthesis. Blackwell, New York

Falkowski PG, Wyman K, Ley AC, Mauzerall DC (1986) Relationship of steady-state photosynthesis to fluorescence in eukaryotic algae. Biochim Biophys Acta 849:183-192

Geider RJ, Osborne BA (1992) Algal photosynthesis: the measurement of algal gas exchange current phycology, Vol 2, Chapman \& Hall, New York

Geider RJ, La Roche J, Greene RM, Olaizola M (1993) Response of the photosynthetic apparatus of Phaeodactylum tricornutum (Bacillariophyceae) to nitrate, phosphate, or iron starvation. J Phycol 29:755-766

Geider RJ, Macintyre HL, Graziano LM, McKay RML (1998) Responses of the photosynthetic apparatus of Dunaliella tertiolecta (Chlorophyceae) to nitrogen and phosphorus limitation. Eur J Phycol 33:315-332

Gibb SW, Barlow RG, Cummings DG, Rees NW, Trees CC, Holligan P, Suggett D (2000) Surface phytoplankton pigment distributions in the Atlantic Ocean: an assessment of basin scale variability between 50 degrees $\mathrm{N}$ and 50 degrees S. Prog Oceanogr 45:339-368

Greene RM, Geider RJ, Kolber Z, Falkowski PG (1992) Ironinduced changes in light harvesting and photochemical energy conversion processes in eukaryotic marine algae. Plant Physiol 100:565-575

Guillard RRL, Ryther JH (1962) Studies of marine planktonic diatoms I. Cyclotella Nana Hustedt, and Detonula confervacea (Cleve) Gran. Can J Microbiol 8:229-239

Johnsen G, Samset O, Granskog L, Sakshaug E (1994) In vivo absorption characteristics in 10 classes of bloom-forming phytoplankton: taxonomic characteristics and responses to photoadaptation by means of discriminant and HPLC analysis. Mar Ecol Prog Ser 105:149-157

Johnson Z (2000) Regulation of marine photosynthetic efficiency by PSII, PhD thesis, Duke University, Durham, NC

Johnson Z, Barber R (2003) The low-light reduction in the quantum yield of photosynthesis: potential errors and biases when calculating the maximum quantum yield. Photosynth Res 75:85-95

Johnson Z, Howd P (2000) Marine photosynthetic perfor-

Editorial responsibility: Otto Kinne (Editor),

Oldendorf/Luhe, Germany mance forcing and periodicity for the Bermuda Atlantic time series, 1989-1995. Deep-Sea Res Part I 47:1485-1512

Johnson Z, Bidigare RR, Goericke R, Marra J, Trees C, Barber RT (2002) Photosynthetic physiology and physicochemical forcing in the Arabian Sea, 1995. Deep-Sea Res Part I 49: 415-436

Kirk JTO (1994) Light and photosynthesis in aquatic ecosystems. Cambridge University Press, NewYork

Kolber Z, Falkowski PG (1993) Use of active fluorescence to estimate phytoplankton photosynthesis in situ. Limnol Oceanogr 38:1646-1665

Kolber Z, Zehr J, Falkowski P (1988) Effects of growth irradiance and nitrogen limitation on photosynthetic energy conversion in PSII. Plant Physiol 88:923-929

Kolber ZS, Prasil O, Falkowski PG (1998) Measurements of variable chlorophyll fluorescence using fast repetition rate techniques - defining methodology and experimental protocols. Biochim Biophys Acta 1367:88-106

Long SP, Humphries S, Falkowski PG (1994) Photoinhibition of photosynthesis in nature. Annu Rev Plant Physiol Plant Mol Biol 45:633-662

Olaizola M, La Roche J, Kolber Z, Falkowski PG (1994) Nonphotochemical fluorescence quenching and the diadinoxanthin cycle in a marine diatom. Photosynth Res 41: $357-370$

Parkhill JP, Maillet G, Cullen JJ (2001) Fluorescence-based maximal quantum yield for PSII as a diagnostic of nutrient stress. J Phycol 37:517-529

Parsons TR, Maita Y, Lalli CM (1984) A manual of chemical and biological methods for seawater analysis,. Permagon Press, Elmsford, NY

Redfield AC (1958) The biological control of chemical factors in the environment. Am Sci 46:205-211

Schofield O, Prézelin BB, Bidigare RR, Smith RC (1993) In situ photosynthetic quantum yield. Correspondence to hydrographic and optical variability within the Southern California Bight. Mar Ecol Prog Ser 93:1-13

Schreiber U, Schliwa U, Bilger W (1986) Continuous recording of photochemical and non-photochemical chlorophyll fluorescence quenching with a new type of modulation fluorometer. Photosynth Res 10:51-62

Schreiber U, Neubauer C, Klughammer C (1989) Devices and methods for room temperature fluorescence analysis. Philos Trans R Soc Lond B323:241-251

van Kooten O, Snel JFH (1990) The use of chlorophyll fluorescence nomenclature in plant stress physiology. Photosynth Res 25:147-150

Vassiliev IR, Prasil O, Wyman KD, Kolber Z, Hanson AK Jr, Prentice JE, Falkowski PG (1994) Inhibition of PSII photochemistry by PAR and UV radiation in natural phytoplankton communities. Photosynth Res 42:51-64

Walker D (1988) The use of the oxygen electrode and fluorescence probes in simple measurements of photosynthesis, Research Institute for Photosynthesis, University of Sheffield, Sheffield

Submitted: March 31, 2003; Accepted: May 25, 2004

Proofs received from author(s): November 12, 2004 